\title{
Reports
}

\section{Utilization of AFFX spike-in control probes to monitor sample identity throughout Affymetrix GeneChip Array processing}

\author{
Michael Walter, Anja Honegger, Rahel Schweizer, Sven Poths, and Michael Bonin \\ Microarray Facility Tübingen, Institute of Human Genetics, University of Tübingen, Tübingen, Germany
}

BioTechniques 48: 371-378 (May 2010) doi 10.2144/000113421

Keywords: Affymetrix GeneChip; microarray hybridization; gene expression profiling; sample tracking; microarray quality control

Supplementary material for this article is available at www.BioTechniques.com/article/113421.

Microarrays evolved from a highly specialized technique into a standard molecular biology method that is widely used for whole-genome gene expression profiling. One of the most important aspects of this method is sample identity, that is, whether the expression profile recorded from an array actually derives from the indicated sample. Several potential steps in the protocol exist where a mix-up of samples may occur. With the increasing size of microarray studies, it is important to ensure that each expression profile is assigned to the correct sample. Errors at this level almost certainly lead to erroneous results and can even cause a complete failure of the microarray study. We developed a system that utilizes probes already present on commercially available Affymetrix arrays to unambiguously correlate the recorded expression profile with the input sample RNA. A set of eight spike-in controls were generated, which can be added to sample RNA in different combinations to generate an "on-chip identifier" that passes through the entire array processing protocol and results in a sample-specific hybridization pattern. This pattern can then be used to monitor whether each array was hybridized with the correct sample. The spike-in controls did not have any negative effect on RNA integrity or any detectable influence on the expression values of the remaining probes on the array; therefore, these controls represent an inexpensive and easily adaptable system to guarantee high-quality results from microarray experiments.

\section{Introduction}

Microarrays evolved from a highly specialized technique into a standard molecular biology method that is widely used for whole-genome gene expression profiling. However, many crucial steps are necessary to obtain meaningful results. Typically, multiple quality-control steps are employed to monitor sample and array quality throughout the entire arrayprocessing protocol and subsequent data analysis $(1,2)$. One of the most important aspects of this quality control, however, is sample identity, that is, whether the expression profile recorded from an array actually derives from the indicated sample. There are several potential steps where a mix-up of samples may occur, for example column purification or array loading, where the entire reaction is transferred between different tubes. With the increasing size of microarray studies, it is important to ensure that each expression profile is assigned to the correct sample. Errors at this level almost certainly lead to erroneous results and can even cause a complete failure of the microarray study. In some cases, there might be some expression features that allow the confirmation of sample identity. Expression of sex-specific transcripts can be used, for example, to confirm the correct gender of the samples. In most cases, however, no such characteristic can be found. Very often this problem is addressed by the use of laboratory information management software (LIMS), which monitors each sample throughout the entire processing and keeps track of each step performed. Low- to mediumthroughput laboratories, however, often have no access to LIMS resources. And even with LIMS support, there is always the factor of human error, which cannot be controlled completely. Thus, we developed a sample tracking system that utilizes probes already present on commercially available Affymetrix arrays to unambiguously correlate the recorded expression profile with the input sample RNA. A set of eight spike-in controls were generated which can be added to sample RNA in different combinations to generate an "on-chip identifier" which passes through the entire array processing protocol and results in a samplespecific hybridization pattern. This pattern can then be used to monitor whether each array was hybridized with the correct sample. The spike-in controls did not have any negative effect on RNA integrity or any detectable influence on the expression values of the remaining probes on the array and thus represent an inexpensive and easily adaptable system to guarantee high-quality microarray results.

\section{Materials and methods}

Construction of identifier constructs Complementary oligonucleotides consisting of a tandem repeat of the sequence of the targeted probe fused to 20 adenosine residues were mixed at a final concen- 
tration of $10 \mu \mathrm{M}$ and phosphorylated with polynucleotide kinase (Promega, Mannheim, Germany). The sequences of all oligonucleotides used are listed in Supplementary Table S1. After addition of $\mathrm{NaCl}$ to a final concentration of $60 \mathrm{mM}$, the oligonucleotides were heated to $96^{\circ} \mathrm{C}$ for $5 \mathrm{~min}$ and then cooled to $25^{\circ} \mathrm{C}$ with a ramp rate of $0.1^{\circ} \mathrm{C} / \mathrm{s}$ in a thermal cycler to generate double-stranded fragments. These fragments also harbor 5 ' overhanging ends compatible with BamHI and HindIII to allow insertion into pBlueskript SK II+ (Agilent, Waldbronn, Germany). Fragments for microarray hybridization were generated by PCR amplification of the insert region of the plasmids with $0.5 \mu \mathrm{M}$ each standard M13 primer (F: GTAAACGACGGCCAGTG; R: GGAAACAGCTATGACCATGA) with $2 \times$ Phusion HF PCR Master Mix (Finnzymes, Espoo, Finland) and $10 \mathrm{pg}$ plasmid DNA as template. After $30 \mathrm{~s}$ of denaturation at $98^{\circ} \mathrm{C}$, fragments were amplified with 35 cycles consisting of 10 $s$ at $98^{\circ} \mathrm{C}, 30 \mathrm{~s}$ at $58^{\circ} \mathrm{C}$, and $30 \mathrm{~s}$ at $72^{\circ} \mathrm{C}$, and then underwent a final extension step at $72^{\circ} \mathrm{C}$ for $5 \mathrm{~min}$. PCR products were purified using Qiagen PCR purification kit (Qiagen, Hilden, Germany) according to the manufacturer's protocol, and concentration was determined on a Nanodrop UV spectrophotometer (Thermo Scientific, Wilmington, DE, USA). Each fragment was incubated with $1 \mu \mathrm{g}$ RNA from human embryonic kidney 293 (HEK) cells at a concentration of 1 pM for $4 \mathrm{~h}$ at $37^{\circ} \mathrm{C}$. RNA was analyzed on an Agilent Bioanalyzer 2100 (Agilent, Waldbronn, Germany) to monitor effects of the fragments on RNA quality.

\section{RNA extraction}

RNA from HEK cells was extracted with Qiagen RNeasy Mini Kit, and rat tissue samples were homogenized in QIAzol reagent (Qiagen). After separation of organic and aqueous phases, the latter was loaded onto a Qiagen RNeasy Midi column to purify the RNA. RNA was quantitated with a Nanodrop UV spectrofluorometer and quality was monitored on an Agilent Bioanalyzer 2100.

\section{Generation of stock solutions} and identifier combinations

To generate any combination of identifier probes, equal amounts of the PCR products were mixed and diluted to a final concentration of $1 \mathrm{ng} / \mu \mathrm{L}$ in water for all probes except lys3_10, which was adjusted to $2 \mathrm{ng} / \mu \mathrm{L}$. This stock solution has to be prepared fresh on a weekly basis. For longer shelf life, adjust concentrations of

A

600

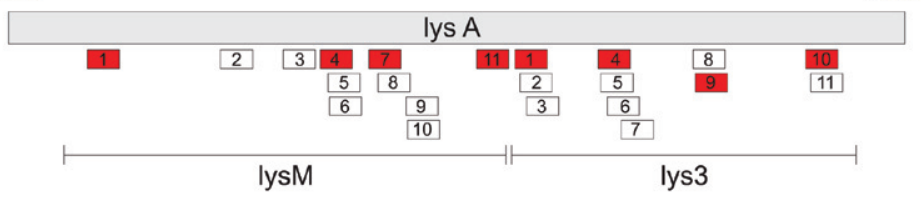

B

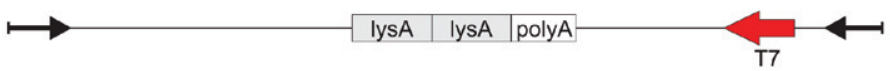

Figure 1. Features from "AFFX-r2-Bs-lys-M_at" and "AFFX-r2-Bs-lys-3_at" probesets were selected for construction of identifier probes. (A) Overview of location of the 11 perfect match probes from the two probe sets "AFFX-r2-Bs-lys-M_at" (lysM) and "AFFX-r2-Bs-lys-3_at" (lys3), respectively. The probe sequences used for constructing identifier fragments are depicted in red. (B) Schematic representation of identifier constructs. Black arrows represent the location of M13 primer used for amplification. The red arrow indicates the T7 promoter. The tandem repeat of the probe sequence is colored light gray; the polyA sequence is represented by the white box.

stocks to $50 \mathrm{ng} / \mu \mathrm{L}$ or add yeast tRNA at a concentration of $100 \mathrm{ng} / \mu \mathrm{L}$. Combined identifier probes were diluted 1:2600 with water and $1 \mu \mathrm{L}$ of this dilution was mixed with $1 \mu \mathrm{g}$ total HEK or rat tissue RNA. A $1 / 10$ th aliquot of this mixture was used as input material for array processing for 3' in vitro transcription ( $3^{\prime}$ IVT) and gene arrays (Affymetrix, Santa Clara, CA).

\section{Microarray processing}

All microarray processing and hybridization steps were carried out according to the recommendations from the manufacturer (Affymetrix, Santa Clara, CA, USA). Briefly, for each hybridization $100 \mathrm{ng}$ of total RNA was used with or without identifier constructs. For 3' IVT arrays (HG-U133 plus 2.0, RAE230A), the Ambion Express Kit (Applied Biosystems, Darmstadt, Germany) was used. For gene arrays on cartridges (HuGene 1.0) and plates (HT-RaGene1.0), RNA was processed with Whole Transcript (WT) Sense Target Labeling Kit and WT Sense Target Labeling Kit 2 (both Affymetrix), respectively. Identifier constructs were added to the total RNA prior to microarray processing at the amounts indicated in the "Results and discussion" section.

\section{Data analysis}

Arrays were scanned using the GCS3000 GeneChip scanner or GeneTitan (both Affymetrix) and AGCC software versions 2.0 and 3.0. Scanned images were subjected to visual inspection to control for hybridization artifacts and proper grid alignment. Raw .CEL files were analyzed with the Bioconductor (www.bioconductor.org) packages 'affy' for 3' IVT arrays (3) and 'xps' for Gene arrays (www. bioconductor.org/packages/release/bioc/ $\mathrm{html} / \mathrm{xps}$.html). Normalization and calculation of signal values were performed with RMA (4). R scripts to extract feature intensities of the targeted control probes and calculate barcodes can be found in the Supplementary Materials.

\section{Results and discussion}

The aim of this study was to evaluate the possibility of using control probes already present on current Affymetrix gene chips as sample tracking devices. Affymetrix arrays harbor a set of probes complementary to bacterial sequences, which can be added to the RNA as polyadenylated transcripts to serve as in-process controls. These transcripts are added in decreasing concentrations to evaluate the sensitivity of the array and to monitor RNA integrity during the in vitro transcription and hybridization process. Our idea was to use the probes of the spike-in control with the lowest concentration, lys A, as targets for additional spike-in controls which should allow sample tracking. Each RNA sample needs a unique combination of control probes. With eight independent probes, a total of 256 combinations are possible, which seems sufficient for most microarray studies. We identified eight nonoverlapping probes in the probe sets "AFFX-r2-Bs-lys-M_at" and "AFFX-r2Bs-lys-3_at" (Figure 1A). These probes are present on all current eukaryotic Affymetrix mRNA expression arrays including gene and exon arrays. Two copies of the sequence of these probes were cloned in tandem into pBlueskript SKII+. This led to higher hybridization signals and increased the size of the fragments added to the RNA, thereby enabling efficient column purification during array processing. A tail of 20 adenosine residues was added. The resulting plasmids can be linearized and used as templates for in vitro transcription resulting in polyadenylated transcripts suitable as spike-in controls. However, the first step of each 
A

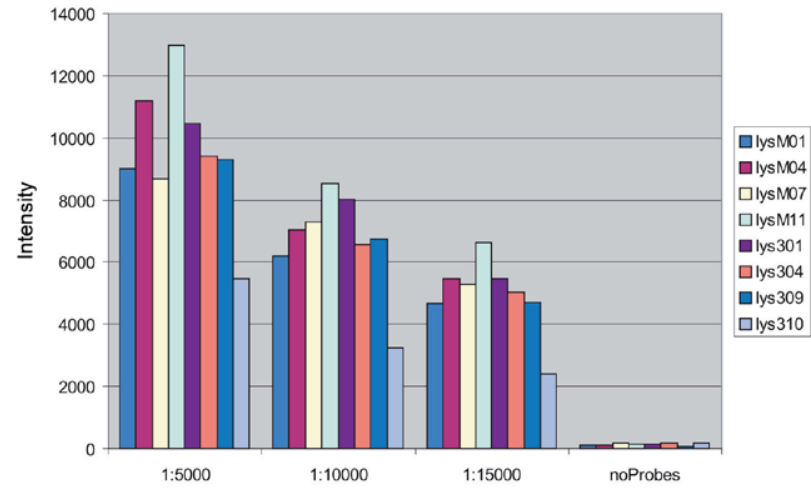

C
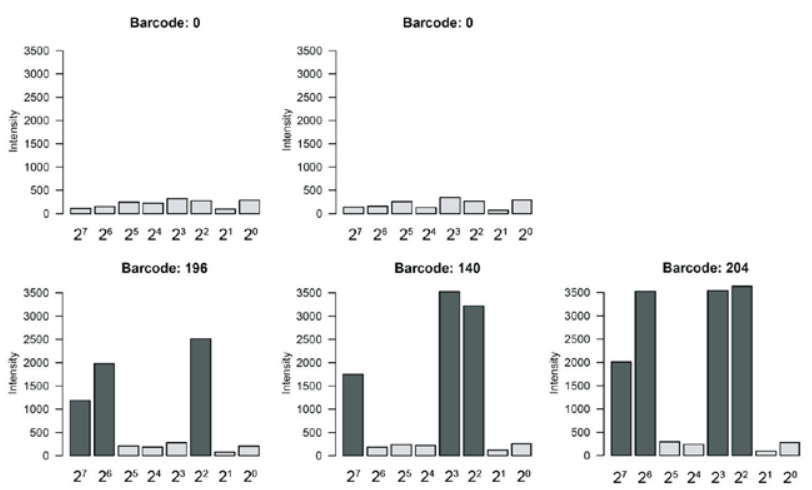

E
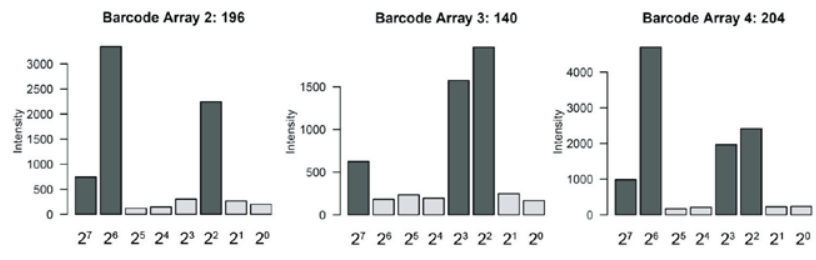

Figure 2. Performance evaluation of identifier probes at different concentrations and combinations. $(A, B)$ Titration of optimal concentration of identifier fragments. (A) Intensities of perfect match probes corresponding to the identifier fragments at the approximate fragment-to-mRNA ratio depicted under the diagram. (B) Pairwise scatterplots and Pearson correlation coefficient of RMA normalized signal intensities from arrays hybridized with HEK RNA and the indicated fragment-to-mRNA ratio. The two probe sets showing large deviations are the probe sets for lysM and lys3. (C,D) Applicability of identifier probes for U133 plus 2.0 arrays. (C) Perfect match intensities of all eight probes chosen for sample tracking recorded on a U133plus 2.0 array. The signals from left to right correspond to lysM 1, 4, 7, 11 and lys3 1, 4, 9, and 10 (see Figure 1A). Combinations of identifier probes are given in the diagonal subpanels of panel D. (D) Pairwise scatterplots and Pearson correlation coefficient of RMA-normalized signal intensities from arrays hybridized with HEK RNA and the indicated identifier combination. (E) Applicability of identifier probes for HuGene 1.0 ST arrays. Perfect match intensities of all eight probes chosen for sample tracking recorded on a HuGene 1.0-ST array. The same combinations of identifier probes as in panels C and D were used.

Affymetrix protocol is to convert RNA into dsDNA harboring a T7 promoter. Therefore, the inserts of the plasmids were simply amplified with standard M13 primer resulting in fragments of 273 nucleotides also including the T7 promoter (Figure 1B). No RNA degradation could be detected when the DNA fragments were incubated with HEK total RNA for $4 \mathrm{~h}$ at $37^{\circ} \mathrm{C}$ (data not shown).

When the Affymetrix polyA spike-in controls were diluted as suggested in the manufacturer's protocol, the average intensity of the probes for lysA was between 100 and 200. The added identifier constructs led to an increase in this intensity. To obtain explicit results, identifier constructs should be added at a concentration that will give rise to signals of 1000 to 5000. Assuming an average RNA size of $1.4 \mathrm{~kb}(5)$ and $2 \%$ mRNA in the total RNA, each microgram of RNA contains $\sim 42 \mathrm{fmol} \mathrm{mRNA}$ molecules. To find the optimal amount of identifier constructs, $1 \mu \mathrm{g}$ HEK total RNA was spiked with 1:5000, 1:10,000, and 1: 15,000 molar ratios of identifier to mRNA molecules and hybridized onto HG-U133 plus 2.0 arrays. This corresponds to a final concentration of 8.4, 4.2, and 2.8 attomoles, respectively, of PCR product per microgram of total RNA. Feature intensities for all eight probes were extracted (Figure 2A). All eight target probes showed an approximately linear response of their signals with decreasing concentrations of added fragments, indicating that all constructs work well and are suitable for sample tracking. With a molar ratio of $1: 15,000$, 


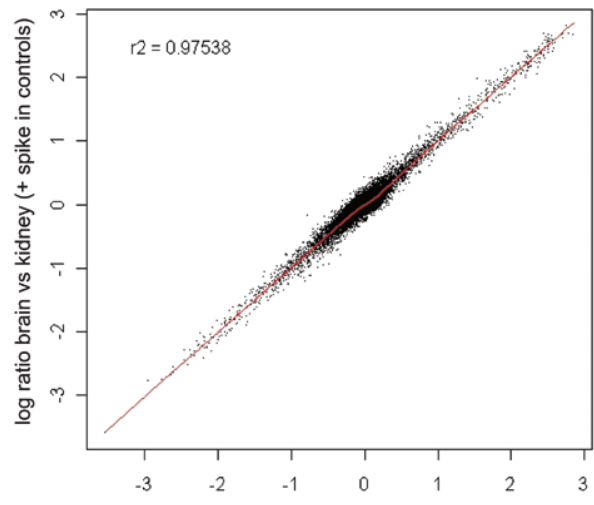

log ratio brain vs kidney (- spike in controls)

Figure 3. The addition of spike-in controls does not affect the expression changes measured on Affymetrix GeneChips. The $\log _{2}$ of the relative changes in expression values of rat liver compared with rat kidney RNA was calculated for samples with or without the addition of identifier fragments and plotted against each other. The Pearson correlation coefficient is depicted in the diagram.

the intensities were approximately at 5000 , which we defined as our upper limit. It was also evident that probe lys3_10 always had only $\sim 50 \%$ of the intensity of the remaining probes. Thus, the optimal concentrations of the identifier constructs are 1:20,000 (2.1 amol/ug RNA), except lys3_10, which should be added at a concentration of 4.8 amol/ $\mu \mathrm{g}$ RNA. When $<1 \mu \mathrm{g}$ RNA is used as starting material, the amount of spike-in constructs has to be adjusted accordingly (see the "Material and methods" section for details). Since the probes are overlapping with other probes from the same probe set (Figure 1), the intensity of the latter will also increase; depending on the number of identifiers added, the overall intensity of the lysA probe sets will change (Figure 2B; Supplementary Figure S1). As a consequence, the lys A signals can no longer be used for quality control. However, the values for the remaining three spike-in RNAs as well as the four hybridization control RNAs will remain unchanged and thus still can be used for quality evaluation (Supplementary Figure S1). One array was hybridized with the same RNA without identifier controls. When the normalized signals of the four arrays were plotted against each other, a high correlation could be observed, which is in the normal range of technical replicates (Figure 2B). Even with all eight constructs spiked in at high concentrations, no effect could be observed on the expression levels of the remaining probe sets.

In a second set of experiments, HEK RNA was spiked with three different combinations of identifier controls and hybridized onto HG-U133 plus 2.0 arrays. Again, the correlation of technical replicates with and without spike-in controls was $>0.99$ (Figure 2, C and D). The intensity threshold was set at 500 and the eight control probes were assigned values of 1 or 0 depending on whether they were above the chosen threshold or not. So the hybridization pattern was translated into an eight-digit binary number which then was retransformed into a number corresponding to the added barcode (Figure 2C). The same RNA-identifier combinations were also hybridized onto $\mathrm{HuGene}$ 1.0 ST arrays. Correlation coefficients of normalized signals were $>0.99$ when RNA with control fragments was compared against RNA without fragments (data not shown). The barcodes could be extracted with the same results as for the U133 plus 2.0 arrays (Figure $2 \mathrm{E}$ ), indicating that the chosen strategy applies for 3' IVT arrays and gene/exon arrays.

The expression profiles of technical replicates are highly reproducible when identifier controls are added. Thus, the expression changes measured on microarrays should be identical with or without the spike-in controls. To test this, we isolated RNA from liver and kidney from two rats. The four samples were hybridized onto RAE230A arrays without controls and after addition of four different identifier combinations. The correlation between technical replicates again was $>0.99$ and correlation of biological replicates was $\sim 0.97$ with or without spike-in fragments (data not shown). Figure 3 shows the log-fold change between liver and kidney samples without controls plotted against the log-fold change from the same comparison with the addition of controls. As expected, the correlation of the log-fold change values was very high, indicating that addition of identifier controls has no impact on the overall performance of the microarray experiment but enables the unambiguous identification of the sample loaded onto each array.

The probe sets "AFFX-r2-Bs-lys-M_at" and "AFFX-r2-Bs-lys-3_at" are universal controls on all current Affymetrix GeneChips. Therefore, the sample tracking strategy outlined here can be used not only for human samples but for any species for which an Affymetrix array is available (e.g., Drosophila, Caenorhabditis elegans, Arabidopsis, etc.). Furthermore, the same strategy can be transferred to other platforms, such as custom-made arrays and other commercially available chips. Illumina human WG6 bead arrays for example, harbor a set of 1374 negative controls used for background subtraction. Here a set of eight probes can be assigned for sample tracking while still leaving the majority of negative controls available. However, when signals of any control probes are manipulated by use of identifier probes, this should be documented when submitting the data to public databases.

The sample tracking system introduced here only covers the experimental procedure after RNA extraction. The system can be extended one step further and the constructs can be added prior to RNA extraction. Since most RNA extraction protocols include a DNase treatment, which would also degrade the PCR products, the constructs must be converted into in vitro-transcribed RNA. Therefore the plasmids harbor the polyA sequence which serves as priming site for cDNA synthesis. However, here the amount of spike-in RNA has to be carefully titrated for each cell line, tissue, etc., to reach the optimal ratio of mRNA:spike-in RNAs.

\section{Acknowledgments}

We greatly appreciate the helpful discussion with Irene Walter and Karina Häbig, and thank Silke Gommel for excellent technical assistance.

\section{Competing interests}

The authors declare no competing interests.

\section{References}

1. Imbeaud, S. and C. Auffray. 2005. 'The 39 steps' in gene expression profiling: critical issues and proposed best practices for microarray experiments. Drug Discov. Today 10:1175-1182.

2. Wilkes, T., H. Laux, and C.A. Foy. 2007. Microarray data quality - review of current developments. OMICS 11:1-13.

3. Gautier, L., L. Cope, B.M. Bolstad, and R.A. Irizarry. 2004. affy--analysis of Affymetrix GeneChip data at the probe level. Bioinformatics 20:307-315.

4.Irizarry, R.A., B. Hobbs, F. Collin, Y.D. Beazer-Barclay, K.J. Antonellis, U. Scherf, and T.P. Speed. 2003. Exploration, normalization, and summaries of high density oligonucleotide array probe level data. Biostatistics 4:249-264.

5. Sommer, S.S. and J.E. Cohen. 1980. The size distributions of proteins, mRNA, and nuclear RNA. J. Mol. Evol. 15:37-57.

Received 22 February 2010; accepted 31 March 2010.

Address correspondence to Michael Walter, Institute of Human Genetics, University of Tübingen, Calwerstrasse 7, D-72076, Tübingen, Germany. e-mail: michael.walter@med.unituebingen.de 